Submitted to Proceedings of ICOMAT 2017

\title{
INTERACTION OF MARTENSITIC MICROSTRUCTURES IN ADJACENT GRAINS
}

\author{
John M. Ball \\ Mathematical Institute, University of Oxford, Woodstock Road, Oxford OX2 6GG, U.K. \\ Carsten Carstensen \\ Department of Mathematics, Humboldt-Universität zu Berlin, Unter den Linden 6, D-10099 Berlin, \\ Germany

\begin{abstract}
It is often observed that martensitic microstructures in adjacent polycrystal grains are related. For example, micrographs of Arlt [1] (one reproduced in [10, p225]) exhibit propagation of layered structures across grain boundaries in the cubic-to-tetragonal phase transformation in $\mathrm{BaTiO}_{3}$. Such observations are related to requirements of compatibility of the deformation at the grain boundary. Using a generalization of the Hadamard jump condition, this is explored in the nonlinear elasticity model of martensitic transformations for the case of a bicrystal with suitably oriented columnar geometry, in which the microstructure in both grains is assumed to involve just two martensitic variants, with a planar or non-planar interface between the grains.
\end{abstract}

Keywords: Bicrystal, compatibility, grain boundary, Hadamard jump condition.

\section{Description of PRoblem}

Consider a bicrystal consisting of two columnar grains $\Omega_{1}=\omega_{1} \times(0, d)$ (grain 1 ), $\Omega_{2}=$ $\omega_{2} \times(0, d)$ (grain 2), where $d>0$ and $\omega_{1}, \omega_{2} \subset \mathbb{R}^{2}$ are bounded Lipschitz domains whose boundaries $\partial \omega_{1}, \partial \omega_{2}$ intersect nontrivially, so that $\partial \omega_{1} \cap \partial \omega_{2}$ contains points in the interior $\omega$ of $\bar{\omega}_{1} \cup \bar{\omega}_{2}$ (see Fig. 1). Let $\Omega=\omega \times(0, d)$. The interface between the grains is the set $\partial \Omega_{1} \cap \partial \Omega_{2} \cap \Omega=\left(\partial \omega_{1} \cap \partial \omega_{2} \cap \omega\right) \times(0, d)$. Since by assumption the boundaries $\partial \omega_{1}, \partial \omega_{2}$ are locally the graphs of Lipschitz functions, and such functions are differentiable almost everywhere, the interface has at almost every point (with respect to area) a well-defined normal $\mathbf{n}(\theta)=(\cos \theta, \sin \theta, 0)$ in the $\left(x_{1}, x_{2}\right)$ plane. We say that the interface is planar if it is contained in some plane $\{\mathbf{x} \cdot \mathbf{n}=k\}$ for a fixed normal $\mathbf{n}$ and constant $k$.
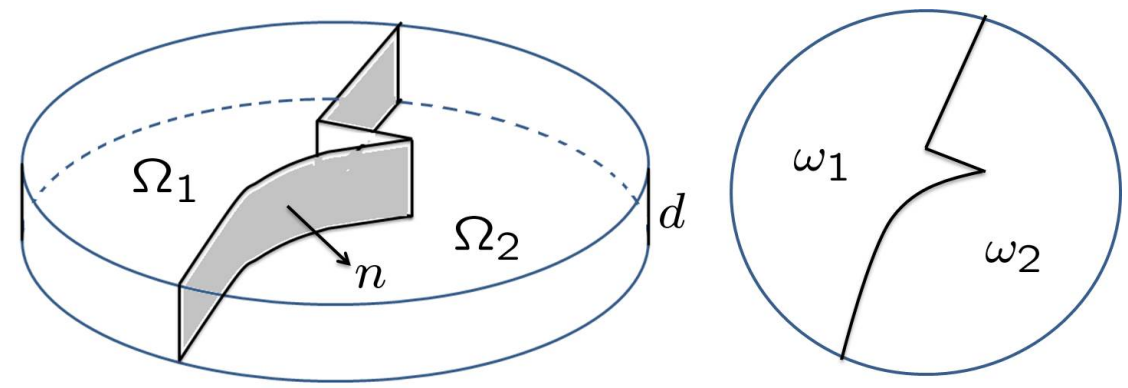

Figure 1. Bicrystal consisting of two grains $\Omega_{1}=\omega_{1} \times(0, d), \Omega_{2}=\omega_{2} \times(0, d)$.

Date: October 3, 2018. 
We use the nonlinear elasticity model of martensitic transformations from [6, 8], with corresponding free-energy density $\psi(\nabla \mathbf{y}, \theta)$ for a single crystal at temperature $\theta$ and deformation $\mathbf{y}=\mathbf{y}(\mathbf{x})$ with respect to undistorted austenite at the critical temperature $\theta_{c}$ at which the austenite and martensite have the same free energy. We denote by $\mathbb{R}_{+}^{n \times n}$ the set of real $n \times n$ matrices $\mathbf{A}$ with $\operatorname{det} \mathbf{A}>0$, and by $S O(n)$ the set of rotations in $\mathbb{R}^{n}$. At a fixed temperature $\theta<\theta_{c}$, we suppose that

$$
K=S O(3) \mathbf{U}_{1} \cup S O(3) \mathbf{U}_{2}
$$

is the set of $\mathbf{A} \in \mathbb{R}_{+}^{3 \times 3}$ minimizing $\psi(\mathbf{A}, \theta)$, where $\mathbf{U}_{1}=\operatorname{diag}\left(\eta_{2}, \eta_{1}, \eta_{3}\right), \mathbf{U}_{2}=\operatorname{diag}\left(\eta_{1}, \eta_{2}, \eta_{3}\right)$ and $\eta_{2}>\eta_{1}>0, \eta_{3}>0$. This corresponds to a tetragonal to orthorhombic phase transformation (see [10, Table 4.6]), or to an orthorhombic to monoclinic transformation in which the transformation strain involves stretches of magnitudes $\eta_{1}, \eta_{2}$ with respect to perpendicular directions lying in the plane of two of the orthorhombic axes and making an angle of $\pi / 4$ with respect to these axes ${ }^{1}$. Alternatively, for example, taking $\eta_{3}=\eta_{1}$ the analysis of this paper can be viewed as applying to a cubic to tetragonal transformation under the a priori assumption that only two variants are involved in the microstructure.

We suppose that $\Omega_{1}$ has cubic axes in the coordinate directions $\mathbf{e}_{1}, \mathbf{e}_{2}, \mathbf{e}_{3}$, while in $\Omega_{2}$ the cubic axes are rotated through an angle $\alpha$ about $\mathbf{e}_{3}$. By adding a constant to $\psi$ we may assume that $\psi(\mathbf{A}, \theta)=0$ for $\mathbf{A} \in K$. Then a zero-energy microstructure corresponds to a gradient Young measure $\mathrm{I}^{2}\left(\nu_{\mathbf{x}}\right)_{\mathbf{x} \in \Omega}$ such that

$$
\operatorname{supp} \nu_{\mathbf{x}} \subset K \text { for a.e. } \mathbf{x} \in \Omega_{1}, \operatorname{supp} \nu_{\mathbf{x}} \subset K \mathbf{R}_{\alpha} \text { for a.e. } \mathbf{x} \in \Omega_{2},
$$

where

$$
\mathbf{R}_{\alpha}=\left(\begin{array}{lll}
\cos \alpha & -\sin \alpha & 0 \\
\sin \alpha & \cos \alpha & 0 \\
0 & 0 & 1
\end{array}\right)
$$

It is easily shown that $K \mathbf{R}_{\alpha}=K$ if and only if $\alpha=n \pi / 2$ for some integer $n$, and that $K \mathbf{R}_{\alpha+\pi / 2}=K \mathbf{R}_{\alpha}$. We thus assume that $0<\alpha<\frac{\pi}{2}$, since this covers all nontrivial cases.

As remarked in [5], by a result from [9] there always exists a zero-energy microstructure constructed using laminates, with gradient Young measure $\nu_{\mathbf{x}}=\nu$ satisfying (1.2) that is independent of $x$ and has macroscopic deformation gradient $\bar{\nu}=\int_{\mathbb{R}_{+}^{3 \times 3}} \mathbf{A} d \nu(\mathbf{A})=\left(\operatorname{det} \mathbf{U}_{1}\right) \mathbf{1}$. Our aim is to give conditions on the deformation parameters $\eta_{1}, \eta_{2}, \eta_{3}$, the rotation angle $\alpha$ and the grain geometry which ensure that any zero-energy microstructure has a degree of complexity in each grain, in the sense that it does not correspond to a pure variant with constant deformation gradient in either of the grains.

\footnotetext{
${ }^{1}$ The general form of the transformation stretch for an orthorhombic to monoclinic transformation is given in [8, Theorem 2.10(4)]. In general one can make a linear transformation of variables in the reference configuration which turns the corresponding energy wells into the form (1.1). However, in [4, Section 4.1] and the announcement of the results of the present paper in [5] it was incorrectly implied that the analysis based on $K$ as in (1.1) applies to a general orthorhombic to monoclinic transformation. This is not the case because the linear transformation in the reference configuration changes the deformation gradient corresponding to austenite in [4] and to the rotated grain in the present paper. A more general, but feasible, analysis would be needed to cover the case of general orthorhombic to monoclinic transformations.

${ }^{2}$ For an explanation of gradient Young measures and how they can be used to represent possibly infinitely fine microstructures see, for example, [2].
} 


\section{RANK-ONE CONNECTIONS BETWEEN ENERGY WELLS}

Let $\mathbf{U}=\mathbf{U}^{T}>0, \mathbf{V}=\mathbf{V}^{T}>0$. We say that the energy wells $\mathrm{SO}(3) \mathbf{U}, \mathrm{SO}(3) \mathbf{V}$ are rank-one connected if there exist $\mathbf{R}, \mathbf{Q} \in S O(3), \mathbf{a}, \mathbf{n} \in \mathbb{R}^{3},|\mathbf{n}|=1$ with $\mathbf{R U}=\mathbf{Q V}+\mathbf{a} \otimes \mathbf{n}$, where without loss of generality we can take $\mathbf{Q}=\mathbf{1}$. By the Hadamard jump condition this is equivalent to the existence of a continuous piecewise affine map $\mathbf{y}$ whose gradient $\nabla \mathbf{y}$ takes constant values $\mathbf{A} \in S O(3) \mathbf{U}$ and $\mathbf{B} \in S O(3) \mathbf{V}$ on either side of a plane with normal n. The following is an apparently new version of a well-known result (see, for example, 6 , Prop. 4], [2, Theorem 2.1], [12]), giving necessary and sufficient conditions for two wells to be rank-one connected. A similar statement was obtained by Mardare [13].

Lemma 1. Let $\mathbf{U}=\mathbf{U}^{T}>0, \mathbf{V}=\mathbf{V}^{T}>0$. Then $\mathrm{SO}(3) \mathbf{U}, \mathrm{SO}(3) \mathbf{V}$ are rank-one connected if and only if

$$
\mathbf{U}^{2}-\mathbf{V}^{2}=\gamma(\mathbf{m} \otimes \mathbf{n}+\mathbf{n} \otimes \mathbf{m})
$$

for unit vectors $\mathbf{m}, \mathbf{n}$ and some $\gamma \neq 0$. For suitable $\mathbf{a}_{1}, \mathbf{a}_{2} \in \mathbb{R}^{3}$ and $\mathbf{R}_{1}, \mathbf{R}_{2} \in \mathrm{SO}(3)$, the rank-one connections between $\mathbf{V}$ and $\mathrm{SO}(3) \mathbf{U}$ are given by

$$
\mathbf{R}_{1} \mathbf{U}=\mathbf{V}+\mathbf{a}_{1} \otimes \mathbf{n}, \quad \mathbf{R}_{2} \mathbf{U}=\mathbf{V}+\mathbf{a}_{2} \otimes \mathbf{m} .
$$

We omit the proof, which is not difficult. The main point of the lemma is that the normals corresponding to the rank-one connections are the vectors appearing in (2.1). An interesting consequence is that if $\mathbf{U}, \mathbf{V}$ correspond to martensitic variants, so that $\mathbf{V}=\mathbf{Q}^{T} \mathbf{U Q}$ for some $\mathbf{Q} \in S O(3)$, then, taking the trace in (2.1) shows that the two possible normals are orthogonal (see [2, Theorem 2.1]).

Using Lemma 1 we can calculate the rank-one connections between $K$ and $K \mathbf{R}_{\alpha}$. For example, for the rank-one connections between $\mathrm{SO}(3) \mathbf{U}_{1}$ and $\mathrm{SO}(3) \mathbf{U}_{1} \mathbf{R}_{\alpha}$ we find that

$$
\mathbf{U}_{1}^{2}-\mathbf{R}_{\alpha}^{T} \mathbf{U}_{1}^{2} \mathbf{R}_{\alpha}=\left(\eta_{2}^{2}-\eta_{1}^{2}\right) \sin \alpha(\mathbf{m} \otimes \mathbf{n}+\mathbf{n} \otimes \mathbf{m})
$$

where $\mathbf{n}=(\sin (\alpha / 2), \cos (\alpha / 2), 0), \mathbf{m}=(\cos (\alpha / 2),-\sin (\alpha / 2), 0)$ so that the two possible normals $\mathbf{n}=\left(n_{1}, n_{2}, 0\right)$ satisfy $\tan \alpha=2 n_{1} n_{2} /\left(n_{2}^{2}-n_{1}^{2}\right)$. Swapping $\eta_{1}$ and $\eta_{2}$ we see that the possible normals for rank-one connections between $\mathrm{SO}(3) \mathbf{U}_{2}$ and $\mathrm{SO}(3) \mathbf{U}_{2} \mathbf{R}_{\alpha}$ are the same. Similarly, we find that the possible normals for rank-one connections between $\mathrm{SO}(3) \mathbf{U}_{1}$ and $\mathrm{SO}(3) \mathbf{U}_{2} \mathbf{R}_{\alpha}$ or between $\mathrm{SO}(3) \mathbf{U}_{2}$ and $\mathrm{SO}(3) \mathbf{U}_{1} \mathbf{R}_{\alpha}$ satisfy $\tan \alpha=\left(n_{1}^{2}-n_{2}^{2}\right) / 2 n_{1} n_{2}$.

\section{MAIN RESULTS}

Suppose there exists a gradient Young measure of the form 1.2 such that $\nu_{\mathbf{x}}=\delta_{\mathbf{F}}$ for a.e. $\mathbf{x} \in \Omega_{1}$ for some $\mathbf{F} \in K$, corresponding to a pure variant in grain 1 . It follows that the corresponding macroscopic gradient $\nabla \mathbf{y}(\mathbf{x})=\bar{\nu}_{\mathbf{x}}=\int_{M_{+}^{3 \times 3}} \mathbf{A} d \nu_{x}(\mathbf{A})$ satisfies

$$
\nabla \mathbf{y}(\mathbf{x})=\mathbf{F} \text { for a.e. } \mathbf{x} \in \Omega_{1}, \quad \nabla \mathbf{y}(\mathbf{x}) \in\left(K \mathbf{R}_{\alpha}\right)^{\mathrm{qc}} \text { for a.e. } \mathbf{x} \in \Omega_{2}
$$

where $E^{\mathrm{qc}}$ denotes the quasiconvexification of a compact set $E \subset \mathbb{R}^{3 \times 3}$, that is the set of possible macroscopic deformation gradients corresponding to microstructures using gradients in E. As determined in [6, Theorem 5.1] (and more conveniently in [10, p155]) $K^{\mathrm{qc}}$ consists of those $\mathbf{A} \in \mathbb{R}_{+}^{3 \times 3}$ with

$$
\mathbf{A}^{T} \mathbf{A}=\left(\begin{array}{ccc}
a & c & 0 \\
c & b & 0 \\
0 & 0 & \eta_{3}^{2}
\end{array}\right)_{3} \text { and }(a, b, c) \in P,
$$




$$
P=\left\{(a, b, c): a>0, b>0, a b-c^{2}=\eta_{1}^{2} \eta_{2}^{2}, a+b+|2 c| \leq \eta_{1}^{2}+\eta_{2}^{2}\right\},
$$

and is equal to the polyconvexification $K^{p c}$ of $K$. It follows that $\left(K \mathbf{R}_{\alpha}\right)^{\mathrm{qc}}=K^{\mathrm{qc}} \mathbf{R}_{\alpha}=$ $K^{\mathrm{pc}} \mathbf{R}_{\alpha}$. The proof of $(3.2)$ shows also that the quasiconvexification $\tilde{K}^{\mathrm{qc}}$ of $\tilde{K}=S O(2) \tilde{\mathbf{U}}_{1} \cup$ $S O(2) \tilde{\mathbf{U}}_{2}$, where $\tilde{\mathbf{U}}_{1}=\left(\begin{array}{cc}\eta_{2} & 0 \\ 0 & \eta_{1}\end{array}\right), \tilde{\mathbf{U}}_{2}=\left(\begin{array}{cc}\eta_{1} & 0 \\ 0 & \eta_{2}\end{array}\right)$, is equal to $\tilde{K}^{\text {pc }}$ and is given by the set of $\tilde{\mathbf{A}} \in \mathbb{R}_{+}^{2 \times 2}$ such that $\tilde{\mathbf{A}}^{T} \tilde{\mathbf{A}}=\left(\begin{array}{ll}a & c \\ c & b\end{array}\right)$ for $(a, b, c) \in P$.

Let $\mathbf{x}_{0}=\left(\tilde{\mathbf{x}}_{0}, \delta\right)$, where $\tilde{\mathbf{x}}_{0} \in \partial \omega_{1} \cap \partial \omega_{2} \cap \omega, 0<\delta<d$, be such that the interface has a well-defined normal $\mathbf{n}=(\cos \theta, \sin \theta, 0)=(\tilde{\mathbf{n}}, 0)$ at $\mathbf{x}_{0}$. By [7], there exists $\varepsilon>0$ such that in $\mathcal{U}:=B\left(\tilde{\mathbf{x}}_{0}, \varepsilon\right) \times(\delta-\varepsilon, \delta+\varepsilon), 0<\varepsilon<\delta$, the map $\mathbf{y}$ is a plane strain, that is

$$
\mathbf{y}(\mathbf{x})=\mathbf{R}\left(z_{1}\left(x_{1}, x_{2}\right), z_{2}\left(x_{1}, x_{2}\right), \eta_{3} x_{3}+\gamma\right) \text { for a.e. } \mathbf{x} \in \mathcal{U}
$$

for some $\mathbf{R} \in S O(3), \mathbf{z}: B\left(\tilde{\mathbf{x}}_{0}, \varepsilon\right) \rightarrow \mathbb{R}^{2}$ and $\gamma \in \mathbb{R}$, where $B\left(\tilde{\mathbf{x}}_{0}, \varepsilon\right)$ denotes the open ball in $\mathbb{R}^{2}$ with centre $\tilde{\mathbf{x}}_{0}$ and radius $\varepsilon$. Without loss of generality we can take $\mathbf{R}=\mathbf{1}$. Then, for $\tilde{\mathbf{x}}=\left(x_{1}, x_{2}\right) \in B\left(\tilde{\mathbf{x}}_{0}, \varepsilon\right)$ we have

$$
\nabla \mathbf{z}(\tilde{\mathbf{x}})=\tilde{\mathbf{F}} \in \tilde{K} \text { for a.e. } \tilde{\mathbf{x}} \in \omega_{1}, \quad \nabla \mathbf{z}(\tilde{\mathbf{x}}) \in\left(\tilde{K} \tilde{\mathbf{R}}_{\alpha}\right)^{\mathrm{pc}}=\tilde{K}^{\mathrm{pc}} \tilde{\mathbf{R}}_{\alpha} \text { for a.e. } \tilde{\mathbf{x}} \in \omega_{2},
$$

where $\tilde{\mathbf{R}}_{\alpha}=\left(\begin{array}{cc}\cos \alpha & -\sin \alpha \\ \sin \alpha & \cos \alpha\end{array}\right)$. By a two-dimensional generalization of the Hadamard jump condition proved in [3] this implies that there exists $\tilde{\mathbf{A}} \in\left(\tilde{K} \tilde{\mathbf{R}}_{\alpha}\right)^{\text {pc }}$ such that

$$
\tilde{\mathbf{F}}-\tilde{\mathbf{A}}=\tilde{\mathbf{a}} \otimes \tilde{\mathbf{n}}
$$

for some $\tilde{\mathbf{a}} \in \mathbb{R}^{2}$. Conversely, if the interface is planar with normal $\mathbf{n}=(\tilde{\mathbf{n}}, 0)$, then the existence of $\tilde{\mathbf{A}} \in\left(\tilde{K} \tilde{\mathbf{R}}_{\alpha}\right)^{\text {pc }}$ satisfying $(3.6)$ implies the existence of a gradient Young measure $\nu=\left(\nu_{\mathbf{x}}\right)_{\mathbf{x} \in \Omega}$ satisfying (3.1). Indeed there then exists a sequence of gradients $\nabla \mathbf{z}^{(j)}$ generating a gradient Young measure $\left(\mu_{\tilde{\mathbf{x}}}\right)_{\tilde{\mathbf{x}} \in \omega}$ such that

$$
\bar{\mu}_{\tilde{\mathbf{x}}}=\tilde{\mathbf{F}} \text { for a.e. } \tilde{\mathbf{x}} \in \omega_{1}, \bar{\mu}_{\tilde{\mathbf{x}}}=\tilde{\mathbf{A}} \text { for a.e. } \tilde{\mathbf{x}} \in \omega_{2},
$$

and then $\nabla \mathbf{y}^{(j)}(\mathbf{x})=\left(\begin{array}{ccc}z_{1,1}^{(j)} & z_{1,2}^{(j)} & 0 \\ z_{2,1}^{(j)} & z_{2,2}^{(j)} & 0 \\ 0 & 0 & \eta_{3}\end{array}\right)$ generates such a gradient Young measure.

It turns out that we can say exactly when it is possible to solve (3.6). Set $\tau:=\eta_{2} / \eta_{1}>1$, $s^{*}=\left(\tau^{4}-1\right) /\left(\tau^{4}+1\right)$ and define for $0 \leq s \leq 1$ the $C^{1}$ convex increasing function

$$
f(s):=\left\{\begin{array}{cl}
\left(\tau^{4}+1-2 \tau^{2} \sqrt{1-s^{2}}\right) /\left(\tau^{4}-1\right) & \text { if } s \leq s^{*} \\
s & \text { if } s>s^{*}
\end{array}\right.
$$

Theorem 2. There exist $\tilde{\mathbf{F}} \in \tilde{K}$ and $\tilde{\mathbf{A}} \in \tilde{K}^{\mathrm{pc}} \mathbf{R}_{\alpha}$ with $\tilde{\mathbf{F}}-\tilde{\mathbf{A}}=\tilde{\mathbf{a}} \otimes \tilde{\mathbf{n}}$ for $\tilde{\mathbf{n}}=(\cos \theta, \sin \theta)$ and some $\tilde{\mathbf{a}} \in \mathbb{R}^{2}$ if and only if

$$
|\cos 2 \theta| \leq f(|\cos 2(\alpha+\theta)|) .
$$

Proof. It is easily checked that the existence of $\tilde{\mathbf{F}} \in S O(3) \tilde{\mathbf{U}}_{i}$ and $\tilde{\mathbf{A}}$ is equivalent to the existence of $(a, b, c) \in P$ such that $\left|\tilde{\mathbf{U}}_{i} \tilde{\mathbf{n}}^{\perp}\right|^{2}=a N_{1}^{2}+b N_{2}^{2}+2 c N_{1} N_{2}$, where $\tilde{\mathbf{n}}^{\perp}=\left(-n_{2}, n_{1}\right)$ and $\mathbf{N}=\left(N_{1}, N_{2}\right)=\tilde{\mathbf{R}}_{\alpha} \tilde{\mathbf{n}}^{\perp}$. That is

$$
\text { either } n_{1}^{2} \eta_{1}^{2}+n_{2}^{2} \eta_{2}^{2} \text { or } n_{2}^{2} \eta_{1}^{2}+n_{1}^{2} \eta_{2}^{2} \in\left[m_{-}(\mathbf{N}), m_{+}(\mathbf{N})\right]
$$


where $m_{ \pm}(\mathbf{N})=\max _{(a, b, c) \in P}\left(a N_{1}^{2}+b N_{2}^{2}+2 c N_{1} N_{2}\right)$. Changing variables to $x=a+b$ and $y=a-b$ we find that $m_{+}(\mathbf{N})=\max _{(x, y) \in P_{2}} \psi_{+}(x, y), m_{-}(\mathbf{N})=\min _{(x, y) \in P_{2}} \psi_{-}(x, y)$, where

$$
\begin{gathered}
2 \psi_{ \pm}(x, y)=x+y\left(N_{1}^{2}-N_{2}^{2}\right) \pm 2\left|N_{1} N_{2}\right| \sqrt{x^{2}-y^{2}-4 \eta_{1}^{2} \eta_{2}^{2}} \\
P_{2}=\left\{(x, y) \in \mathbb{R}^{2}:|y| \leq \eta_{2}^{2}-\eta_{1}^{2} \text { and } \sqrt{y^{2}+4 \eta_{1}^{2} \eta_{2}^{2}} \leq x \leq \frac{y^{2}+4 \eta_{1}^{2} \eta_{2}^{2}+\left(\eta_{1}^{2}+\eta_{2}^{2}\right)^{2}}{2\left(\eta_{1}^{2}+\eta_{2}^{2}\right)}\right\} .
\end{gathered}
$$

The region $P_{2}$ is bounded by the two arcs $C_{1}:=\left\{x=\sqrt{y^{2}+4 \eta_{1}^{2} \eta_{2}^{2}}\right\}$ and $C_{2}:=\{x=$ $\left.\left(y^{2}+4 \eta_{1}^{2} \eta_{2}^{2}+\left(\eta_{1}^{2}+\eta_{2}^{2}\right)^{2}\right) /\left(2\left(\eta_{1}^{2}+\eta_{2}^{2}\right)\right)\right\}$, defined for $|y| \leq \eta_{2}^{2}-\eta_{1}^{2}$, which intersect at the points $(x, y)=\left(\eta_{1}^{2}+\eta_{2}^{2}, \pm\left(\eta_{2}^{2}-\eta_{1}^{2}\right)\right)$. Note that $\psi_{ \pm}(x, y)$ have no critical points in the interior of $P_{2}$. In fact it is immediate that $\nabla \psi_{+}$cannot vanish, while $\nabla \psi_{-}(x, y)=0$ leads to $y=x\left(N_{2}^{2}-N_{1}^{2}\right)$ and hence to the contradiction $0=x^{2}-y^{2}-4 x^{2} N_{1}^{2} N_{2}^{2}=4 \eta_{1}^{2} \eta_{2}^{2}>0$. Thus the maximum and minimum of $\psi_{ \pm}(x, y)$ are attained on either $C_{1}$ or $C_{2}$. After some calculations we obtain

$$
\begin{aligned}
& m_{+}(\mathbf{N})=\frac{1}{2}\left(\eta_{1}^{2}+\eta_{2}^{2}+\left(\eta_{2}^{2}-\eta_{1}^{2}\right)\left|N_{1}^{2}-N_{2}^{2}\right|\right), \\
& m_{-}(\mathbf{N})= \begin{cases}\frac{\eta_{1}^{2} \eta_{2}^{2}}{\eta_{1}^{2}+\eta_{2}^{2}}\left(1+\sqrt{1-\left|N_{1}^{2}-N_{2}^{2}\right|^{2}}\right) & \text { if }\left|N_{1}^{2}-N_{2}^{2}\right| \leq s^{*}, \\
\frac{1}{2}\left(\eta_{1}^{2}+\eta_{2}^{2}-\left|N_{1}^{2}-N_{2}^{2}\right|\left(\eta_{2}^{2}-\eta_{1}^{2}\right)\right) & \text { if }\left|N_{1}^{2}-N_{2}^{2}\right| \geq s^{*} .\end{cases}
\end{aligned}
$$

Noting that $m_{-}(\mathbf{N}) \leq \frac{1}{2}\left(\eta_{1}^{2}+\eta_{2}^{2}\right) \leq m_{+}(\mathbf{N})$, that $m_{+}(\mathbf{N})+m_{-}(\mathbf{N}) \leq \eta_{1}^{2}+\eta_{2}^{2}$, and that $\frac{1}{2}\left(n_{1}^{2} \eta_{1}^{2}+n_{2}^{2} \eta_{2}^{2}\right)+\frac{1}{2}\left(n_{2}^{2} \eta_{1}^{2}+n_{1}^{2} \eta_{2}^{2}\right)=\frac{1}{2}\left(\eta_{1}^{2}+\eta_{2}^{2}\right)$ it follows that 3.10 is equivalent to

$$
m_{-}(\mathbf{N}) \leq \min \left\{n_{1}^{2} \eta_{1}^{2}+n_{2}^{2} \eta_{2}^{2}, n_{2}^{2} \eta_{1}^{2}+n_{1}^{2} \eta_{2}^{2}\right\}=\frac{1}{2}\left(\eta_{1}^{2}+\eta_{2}^{2}\right)-\frac{1}{2}\left|n_{2}^{2}-n_{1}^{2}\right|\left(\eta_{2}^{2}-\eta_{1}^{2}\right) .
$$

With the relations $n_{1}^{2}-n_{2}^{2}=\cos 2 \theta$ and $N_{2}^{2}-N_{1}^{2}=\cos 2(\theta+\alpha)$ this gives $(3.9)$.

Theorem 3. If the interface between the grains is planar then there always exists a zeroenergy microstructure which is a pure variant in one of the grains.

Proof. The case of a pure variant in grain 2 and a zero-energy microstructure in grain 1 corresponds to replacing $\theta$ by $\theta+\alpha$ and $\alpha$ by $-\alpha$ in the above. Hence, since $f(s) \geq s$, if the conclusion of the theorem were false, Theorem 2 would imply that $|\cos 2 \theta|>f(\mid \cos 2(\alpha+$ $\theta) \mid) \geq|\cos 2(\theta+\alpha)|>f(|\cos 2 \theta|) \geq|\cos 2 \theta|$, a contradiction.

Thus to rule out having a pure variant in one grain the interface cannot be planar.

Theorem 4. There is no zero-energy microstructure which is a pure variant in one of the grains if the interface between the grains has a normal $\mathbf{n}^{(1)}=\left(\cos \theta_{1}, \sin \theta_{1}, 0\right) \in E_{1}$ and a normal $\mathbf{n}^{(2)}=\left(\cos \theta_{2}, \sin \theta_{2}, 0\right) \in E_{2}$, for the disjoint open sets

$E_{1}=\{\theta \in \mathbb{R}: f(|\cos 2(\theta+\alpha)|)<|\cos 2 \theta|\}, \quad E_{2}=\{\theta \in \mathbb{R}: f(|\cos (2 \theta)|)<|\cos 2(\theta+\alpha)|\}$.

Proof. This follows immediately from Theorem 2 and the preceding discussion.

In the case $\alpha=\pi / 4$ the sets $E_{1}, E_{2}$ take a simple form (note that the normals not in $E_{1} \cup E_{2}$ correspond to the rank-one connections between the wells found in Section 2). 
Theorem 5. Let $\alpha=\pi / 4$ and suppos $£^{3}$ that $\eta_{2}^{2} / \eta_{1}^{2}<1+\sqrt{2}$. Then

$$
E_{1}=\bigcup_{j}((4 j-1) \pi / 8,(4 j+1) \pi / 8), \quad E_{2}=\bigcup_{j}((4 j+1) \pi / 8,(4 j+3) \pi / 8) .
$$

Proof. Note that $\eta_{2}^{2} / \eta_{1}^{2}<1+\sqrt{2}$ if and only if $s^{*}<1 / \sqrt{2}$. Therefore if $|\sin 2 \theta|<1 / \sqrt{2}$ then $f(|\sin 2 \theta|)<f(1 / \sqrt{2})=1 / \sqrt{2}<|\cos 2 \theta|$. Hence $f(|\sin 2 \theta|)<|\cos 2 \theta|$ if and only if $|\sin 2 \theta|<1 / \sqrt{2}$. This holds if and only if $\theta \in(\pi / 2) \mathbb{Z}+(-\pi / 8, \pi / 8)=E_{1}$. The case of $E_{2}$ is treated similarly.

\section{Discussion}

Compatibility across grain boundaries in polycrystals using a linearized elastic theory is discussed in [11], [10, Chapter 13]. Whereas we use the nonlinear theory we are restricted to a very special assumed geometry and phase transformation. Nevertheless we are able to determine conditions allowing or excluding a pure variant in one of the grains without any a priori assumption on the microstructure (which could potentially, for example, have a fractal structure near the interface). This was possible using a generalized Hadamard jump condition from [3]. The restriction to a two-dimensional situation is due both to the current unavailability of a suitable three-dimensional generalization of such a jump condition, and because the quasiconvexification of the martensitic energy wells is only known for two wells.

Acknowledgement. The research of JMB was supported by the EU (TMR contract FMRX - CT EU98-0229 and ERBSCI**CT000670), by EPSRC (GRlJ03466, EP/E035027/1, and EP/J014494/1), the ERC under the EU's Seventh Framework Programme (FP7/2007-2013) / ERC grant agreement no 291053 and by a Royal Society Wolfson Research Merit Award.

\section{REFERENCES}

[1] G. Arlt. Twinning in ferroelectric and ferroelastic ceramics: stress relief. J. Materials Science, 22:26552666, 1990.

[2] J. M. Ball. Mathematical models of martensitic microstructure. Materials Science and Engineering A, 78(1-2):61-69, 2004.

[3] J. M. Ball and C. Carstensen. Hadamard's compatibility condition for microstructures. In preparation.

[4] J. M. Ball and C. Carstensen. Compatibility conditions for microstructures and the austenite-martensite transition. Materials Science E Engineering A, 273-275:231-236, 1999.

[5] J. M. Ball and C. Carstensen. Geometry of polycrystals and microstructure. MATEC Web of Conferences, 33:02007, 2015.

[6] J. M. Ball and R. D. James. Fine phase mixtures as minimizers of energy. Arch. Ration. Mech. Anal., 100:13-52, 1987.

[7] J. M. Ball and R. D. James. A characterization of plane strain. Proc. Roy. Soc. London A, 432:93-99, 1991.

[8] J. M. Ball and R. D. James. Proposed experimental tests of a theory of fine microstructure, and the two-well problem. Phil. Trans. Roy. Soc. London A, 338:389-450, 1992.

[9] K. Bhattacharya. Self-accommodation in martensite. Arch. Ration. Mech. Anal., 120:201-244, 1992.

[10] K. Bhattacharya. Microstructure of Martensite. Oxford University Press, 2003.

[11] K. Bhattacharya and R. V. Kohn. Elastic energy minimization and the recoverable strain of polycrystalline shape-memory materials. Arch. Ration. Mech. Anal., pages 99-180, 1997.

[12] A. G. Khachaturyan. Theory of Structural Transformations in Solids. John Wiley, 1983.

[13] S. Mardare. Personal communication.

\footnotetext{
${ }^{3}$ This extra condition, typically satisfied in practice, was accidentally omitted in the announcement in [5].
} 